\title{
Cosmic shear statistics and cosmology ${ }^{\star}$
}

\author{
L. Van Waerbeke ${ }^{1,2}$, Y. Mellier ${ }^{1,3}$, M. Radovich ${ }^{4,1}$, E. Bertin ${ }^{1,3}$, M. Dantel-Fort ${ }^{3}$, H. J. McCracken ${ }^{5}$ \\ O. Le Fèvre ${ }^{5}$, S. Foucaud ${ }^{5}$, J.-C. Cuillandre ${ }^{6,7}$, T. Erben ${ }^{1,3,8}$, B. Jain ${ }^{9,10}$, \\ P. Schneider ${ }^{11}$, F. Bernardeau ${ }^{12}$, and B. Fort ${ }^{1}$ \\ 1 Institut d'Astrophysique de Paris, 98bis boulevard Arago, 75014 Paris, France \\ 2 Canadian Institut for Theoretical Astrophysics, 60 St Georges Str., Toronto, M5S 3H8 Ontario, Canada \\ 3 Observatoire de Paris, DEMIRM, 61 avenue de l'Observatoire, 75014 Paris, France \\ 4 Osservatorio Astronomico di Capodimonte, via Moiariello, 80131m Napoli, Italy \\ 5 Laboratoire d'Astrophysique de Marseille, 13376 Marseille Cedex 12, France \\ 6 Canada-France-Hawaii-Telescope, PO Box 1597, Kamuela, Hawaii 96743, USA \\ 7 Observatoire de Paris, 61 avenue de l'Observatoire, 75014 Paris, France \\ 8 Max Planck Institut für Astrophysiks, Karl-Schwarzschild-Str. 1, Postfach 1523, 85740 Garching, Germany \\ 9 Dept of Physics and Astronomy, University of Pennsylvania, Philadelphia, PA 19104, USA \\ 10 Dept. of Physics and Astronomy, Johns Hopkins University, Baltimore, MD 21218, USA \\ 11 Universitaet Bonn, Auf dem Hügel 71, 53121 Bonn, Germany \\ 12 Service de Physique Théorique, CE de Saclay, 91191 Gif-sur-Yvette Cedex, France
}

Received 2 February 2001 / Accepted 25 May 2001

\begin{abstract}
We report a measurement of cosmic shear correlations using an effective area of $6.5 \mathrm{deg}^{2}$ of the VIRMOS deep imaging survey in progress at the Canada-France-Hawaii Telescope. We measured various shear correlation functions, the aperture mass statistic and the top-hat smoothed variance of the shear with a detection significance exceeding $12 \sigma$. We present results on angular scales from 3 arcsec to half a degree. The lensing origin of the signal is confirmed through tests that rely on the scalar nature of the gravitational potential. The different statistical measures give consistent results over the full range of angular scales. These important tests of the measurements demonstrate that the measured correlations could provide accurate constraints on cosmological parameters, subject to the systematic uncertainty in the source redshift distribution. The measurement over more than two decades of scale allows one to evaluate the effect of the shape of the power spectrum on cosmological parameter estimation. The degeneracy on $\sigma_{8}-\Omega_{0}$ can be broken if priors on the shape of the linear power spectrum (parameterized by $\Gamma$ ) are assumed. For instance, with $\Gamma=0.21$ and at the $95 \%$ confidence level, we obtain $0.65<\sigma_{8}<1.2$ and $0.22<\Omega_{0}<0.55$ for open models, and $\sigma_{8}>0.7$ and $\Omega_{0}<0.4$ for flat $(\Lambda$-CDM) models. We discuss how these results would scale if the assumed source redshift distribution needed to be modified with forthcoming measurements of photometric redshifts. From the tangential/radial mode decomposition we can set an upper limit on the intrinsic shape alignment, which has recently been suggested as a possible contribution to the lensing signal. Within the error bars, there is no detection of intrinsic shape alignment for scales larger than $1^{\prime}$.
\end{abstract}

Key words. cosmology: theory - dark matter - gravitational lensing - large-scale structure of the Universe

\section{Introduction}

Cosmological gravitational lensing produced by large-scale structure (or cosmic shear) has been advocated as a powerful tool to probe the mass distribution in the universe (see the reviews from Mellier 1999; Bartelmann \& Schneider

Send offprint requests to: L. Van Waerbeke, e-mail: waerbeke@iap.fr

* Based on observations obtained at the Canada-FranceHawaii Telescope (CFHT) which is operated by the National Research Council of Canada (NRCC), the Institut des Sciences de l'Univers (INSU) of the Centre National de la Recherche Scientifique (CNRS) and the University of Hawaii (UH).
2001 and references therein). The first detections reported over the past year (Van Waerbeke et al. 2000; Bacon et al. 2000; Kaiser et al. 2000; Wittman et al. 2000; Maoli et al. 2001; Rhodes et al. 2001) confirmed that the amplitude and the shape of the signal are compatible with theoretical expectations, although the data sets were not large enough to place strong constraints on cosmological models. Maoli et al. (2001) combined the results from different groups to obtain constraints on the power spectrum normalization $\sigma_{8}$ and the mean density of the universe $\Omega_{0}$ : Their result is in agreement with the cluster abundance constraints, but they were not yet able to break the degeneracy between $\sigma_{8}$ and $\Omega_{0}$. 
The physical interpretation of the weak lensing signal can be made more securely using detections of cosmic shear from different statistics and angular scales on the same data set (as in Van Waerbeke et al. 2000). Unfortunately, their joint detection of the variance and the correlation function using the same data was not fully conclusive: the sample was too small to enable a significant detection of the cosmic shear from variances with different weighting schemes and 2-point statistics over a wide range of scales. The use of independent approaches is nevertheless necessary and it is an important step to validate the reliability of cosmic shear, to check the consistency of the measurements against theoretical predictions and to understand the residual systematics. A relevant example is the aperture mass statistic (defined in Schneider et al. 1998). It is a direct probe of the projected mass power spectrum, and it is not sensitive to certain type of systematics (like a uniform PSF anisotropy) which may corrupt the top-hat smoothed variance, or the shear correlation function. Even the shear correlation function can be measured in several ways, by splitting for instance the tangential and radial modes.

In this paper we report the measurement of the top-hat smoothed variance, the aperture mass, the shear correlation function, and the tangential and radial shear correlation functions on a new homogeneous data set covering an effective area of $6.5 \mathrm{deg}^{2}$. The depth and the field of view are well suited for a comprehensive analysis using various statistics. We show that the amplitude of residual systematics is very low compared to the signal and discuss the consistency of these measurements against the predictions of cosmological models.

We also discuss alternative interpretations. It has been suggested recently that intrinsic alignments of galaxies caused by tidal fields could contribute to the lensing signal (Pen et al. 2000; Croft \& Metzler 2000; Heavens et al. 2000; Catelan et al. 2000; Crittenden et al. 2000a; Crittenden et al. 2000b). This type of systematic is problematic because its signature on different 2-points statistics mimics the lensing effect. A mode decomposition in electric and magnetic types (or $E$ and $B$ modes), similar to what is performed for the polarization analysis in the Cosmic Microwave Background, can separate lensing from intrinsic alignment (see Crittenden et al. 2000a; Crittenden et al. 2000b). The $E$ and $B$ mode analysis is the subject of a forthcoming paper; the aperture mass statistic presented in this paper is a similar analysis to the $E$ and $B$ mode decomposition, and allows us to put an upper limit on the contamination of our survey by the intrinsic alignments.

This paper is organized as follow: Sect. 2 describes our data set, and highlights the differences in the data preprocessing from our previous analysis (Van Waerbeke et al. 2000). The measurement of the shear from this imaging data is discussed in Sect. 3. Section 4 summarizes the theoretical aspects of the different quantities we measure, and lists the statistical estimators used. The results and comparison to a few standard cosmological models are shown in Sect. 5. In Sect. 6 we perform a maximum likelihood analysis of cosmological models in the $\left(\Omega_{0}, \sigma_{8}\right)$ parameter space. The results on very small scales are shown separately in Sect. 7, and we conclude in Sect. 8.

\section{The data set}

The DESCART weak lensing project ${ }^{1}$ is a theoretical and observational program for cosmological weak lensing investigations. The cosmic shear survey carried out by the DESCART team uses the CFH12K data jointly with the VIRMOS survey ${ }^{2}$ to produce a large homogeneous photometric sample which will eventually contain a catalog of galaxies with redshifts as well as the projected mass density over the whole field (Le Fèvre et al. 2001). In contrast to Van Waerbeke et al. (2000), the new sample presented in this work only uses I-band data taken with the $\mathrm{CFH} 12 \mathrm{~K}$ camera and is therefore more homogeneous. It is worth noting that only half of the data of the previous CFHT12K sample is reused in our new sample. A comparison of the results will also allow checking the consistency and the robustness of the cosmic shear analysis.

The CFH12K data was obtained during dark nights in May 1999, November 1999 and April 2000 following the standard observation procedure described in Van Waerbeke et al. (2000). The fields are spread over 4 independent $2 \times 2 \operatorname{deg}^{2}$ areas of the sky identified as F02, F10, F14 and F22. Each field is a compact mosaic of $16 \mathrm{CFH} 12 \mathrm{~K}$ pointings named $\mathrm{P}[\mathrm{n}]$ with $n=1-16$. Once the survey is completed, each of them will cover $4 \mathrm{deg}^{2}$. Currently, of the final $16 \mathrm{deg}^{2}$, only $8.38 \mathrm{deg}^{2}$ is available for the analysis - most of the pointings are located in three different fields (F02, F10, F14 listed in Table 1). This total field of view gets significantly reduced by the masking and selection procedures described below. A summary of the data set characteristics are listed in Table 1.

The data reduction was done at the TERAPIX data center $^{3}$. More than 1.5 Tbytes of data were processed in order to produce the final stacked images. The reduction procedure is the same as in Van Waerbeke et al. (2000), so we refer the reader to this paper for the details. However, in order to improve the image quality prior to correction for the PSF anisotropy and to get a better signal-to-noise ratio on a larger angular scale than in our previous work, all CFH12K images were co-added after astrometric corrections.

The astrometric calibration and the co-addition were done using the MSCRED package in IRAF. Some tasks have been modified in order to allow a fully automatic usage of the package. For each pointing, we first started with the images in the $I$ band. An astrometric solution was first found for one set of exposures in the dither sequence using the USNO-A 2.0 as reference, which provides the position of $\sim \times 10^{8}$ sources with an RMS accuracy of 0.3 arcsec (that is $300-500$ objects per field).

\footnotetext{
1 http://terapix.iap.fr/Descart

2 http://www.astrsp-mrs.fr/virmos/

3 http://terapix.iap.fr
} 
Table 1. List of the fields. All observations were done in $I$ band with the CFH12K camera (Cuillandre et al.2000). The number following the $\mathrm{F}$ denotes the field name, and the number following the $\mathrm{P}$ denotes the pointing name within the field. The geometry of the survey is detailed in http://terapix.iap.fr/Descart/. The image quality has been measured on each stacked image from a standard fitting of a Moffat profile.

\begin{tabular}{|c|c|c|c|c|}
\hline Target & Used area & Exp. time & Period & Image quality \\
\hline F02P1 & $980 \operatorname{arcmin}^{2}$ & $9390 \mathrm{~s}$ & Nov. 1999 & $0.75^{\prime \prime}$ \\
\hline $\mathrm{F} 02 \mathrm{P} 2$ & $1078 \operatorname{arcmin}^{2}$ & $7200 \mathrm{~s}$ & Nov. 1999 & $0.90^{\prime \prime}$ \\
\hline F02P3 & $980 \operatorname{arcmin}^{2}$ & $7200 \mathrm{~s}$ & Nov. 1999 & $0.90^{\prime \prime}$ \\
\hline $\mathrm{F} 02 \mathrm{P} 4$ & $1078 \operatorname{arcmin}^{2}$ & $7200 \mathrm{~s}$ & Nov. 1999 & $0.80^{\prime \prime}$ \\
\hline F10P1 & $882 \operatorname{arcmin}^{2}$ & $3600 \mathrm{~s}$ & May 1999 & $0.65^{\prime \prime}$ \\
\hline F10P2 & $882 \operatorname{arcmin}^{2}$ & $3600 \mathrm{~s}$ & May 1999 & $0.75^{\prime \prime}$ \\
\hline F10P3 & $490 \operatorname{arcmin}^{2}$ & $3600 \mathrm{~s}$ & May 1999 & $0.75^{\prime \prime}$ \\
\hline F10P4 & $882 \operatorname{arcmin}^{2}$ & $3600 \mathrm{~s}$ & May 1999 & $0.65^{\prime \prime}$ \\
\hline F10P5 & $882 \operatorname{arcmin}^{2}$ & $3600 \mathrm{~s}$ & May 1999 & $0.75^{\prime \prime}$ \\
\hline F10P7 & $1176 \operatorname{arcmin}^{2}$ & $3600 \mathrm{~s}$ & Apr. 2000 & $0.75^{\prime \prime}$ \\
\hline F10P8 & $1176 \operatorname{arcmin}^{2}$ & $3600 \mathrm{~s}$ & Apr. 2000 & $0.70^{\prime \prime}$ \\
\hline F10P9 & $98 \operatorname{arcmin}^{2}$ & $3600 \mathrm{~s}$ & Apr. 2000 & $0.65^{\prime \prime}$ \\
\hline F10P10 & $784 \operatorname{arcmin}^{2}$ & $3600 \mathrm{~s}$ & Nov. 1999 & $0.80^{\prime \prime}$ \\
\hline F10P11 & $294 \operatorname{arcmin}^{2}$ & $3600 \mathrm{~s}$ & Nov. 1999/Apr. 2000 & $0.90^{\prime \prime}$ \\
\hline $\mathrm{F} 10 \mathrm{P} 12$ & $1176 \operatorname{arcmin}^{2}$ & $3600 \mathrm{~s}$ & Apr. 2000 & $0.80^{\prime \prime}$ \\
\hline $\mathrm{F} 10 \mathrm{P} 15$ & $686 \operatorname{arcmin}^{2}$ & $3600 \mathrm{~s}$ & Apr. 2000 & $0.85^{\prime \prime}$ \\
\hline F14P1 & $882 \operatorname{arcmin}^{2}$ & $3600 \mathrm{~s}$ & May 1999 & $0.80^{\prime \prime}$ \\
\hline $\mathrm{F} 14 \mathrm{P} 2$ & $882 \operatorname{arcmin}^{2}$ & $3600 \mathrm{~s}$ & May 1999 & $0.85^{\prime \prime}$ \\
\hline F14P3 & $686 \operatorname{arcmin}^{2}$ & $3600 \mathrm{~s}$ & May 1999 & $0.75^{\prime \prime}$ \\
\hline $\mathrm{F} 14 \mathrm{P} 4$ & $1078 \operatorname{arcmin}^{2}$ & $3600 \mathrm{~s}$ & May 1999 & $0.75^{\prime \prime}$ \\
\hline F14P5 & $980 \operatorname{arcmin}^{2}$ & $3600 \mathrm{~s}$ & May 1999 & $0.70^{\prime \prime}$ \\
\hline F14P6 & $686 \operatorname{arcmin}^{2}$ & $3600 \mathrm{~s}$ & May 1999 & $0.80^{\prime \prime}$ \\
\hline F14P7 & $686 \operatorname{arcmin}^{2}$ & $3600 \mathrm{~s}$ & May 1999 & $0.70^{\prime \prime}$ \\
\hline F14P8 & $882 \operatorname{arcmin}^{2}$ & $3600 \mathrm{~s}$ & May 1999 & $0.85^{\prime \prime}$ \\
\hline F14P9 & $1078 \operatorname{arcmin}^{2}$ & $3600 \mathrm{~s}$ & Apr. 2000 & $0.75^{\prime \prime}$ \\
\hline F14P10 & $784 \operatorname{arcmin}^{2}$ & $3600 \mathrm{~s}$ & May 1999 & $0.85^{\prime \prime}$ \\
\hline $\mathrm{F} 14 \mathrm{P} 11$ & $882 \operatorname{arcmin}^{2}$ & $3600 \mathrm{~s}$ & Apr. 2000 & $0.80^{\prime \prime}$ \\
\hline $\mathrm{F} 14 \mathrm{P} 12$ & $784 \operatorname{arcmin}^{2}$ & $3600 \mathrm{~s}$ & Apr. 2000 & $0.80^{\prime \prime}$ \\
\hline F14P13 & $882 \operatorname{arcmin}^{2}$ & $3600 \mathrm{~s}$ & Apr. 2000 & $0.85^{\prime \prime}$ \\
\hline F14P14 & $882 \operatorname{arcmin}^{2}$ & $3600 \mathrm{~s}$ & May 1999 & $1.0^{\prime \prime}$ \\
\hline F14P15 & $882 \operatorname{arcmin}^{2}$ & $3600 \mathrm{~s}$ & Apr. 2000 & $0.90^{\prime \prime}$ \\
\hline F14P16 & $1176 \operatorname{arcmin}^{2}$ & $2880 \mathrm{~s}$ & Apr. 2000 & $0.65^{\prime \prime}$ \\
\hline $\mathrm{F} 22 \mathrm{P} 3$ & $686 \operatorname{arcmin}^{2}$ & $3600 \mathrm{~s}$ & May 1999 & $0.75^{\prime \prime}$ \\
\hline $\mathrm{F} 22 \mathrm{P} 4$ & $980 \operatorname{arcmin}^{2}$ & $3600 \mathrm{~s}$ & Nov. 1999 & $0.75^{\prime \prime}$ \\
\hline $\mathrm{F} 22 \mathrm{P} 6$ & $588 \operatorname{arcmin}^{2}$ & $3600 \mathrm{~s}$ & Apr. 2000 & $0.80^{\prime \prime}$ \\
\hline $\mathrm{F} 22 \mathrm{P} 11$ & $294 \operatorname{arcmin}^{2}$ & $2880 \mathrm{~s}$ & Apr. 2000 & $0.75^{\prime \prime}$ \\
\hline
\end{tabular}

The astrometric solution was then transferred to the other exposures in the sequence. All object catalogs were obtained using SExtractor (Bertin \& Arnouts 1996) ${ }^{4}$ and a linear correction to the world coordinate system was computed with respect to the initial set. Finally, all images were resampled using a bi-cubic interpolation and then stacked together.

At this stage, each stacked image was inspected by eye and all areas which may potentially influence the later lensing analysis signal were masked (see Van Waerbeke et al. 2000 and Maoli et al. 2001). Since we adopted conservative masks, this process had a dramatic impact on the field of view: we lost $20 \%$ of the total area and ended up with a usable area of $6.5 \mathrm{deg}^{2}$.

The photometric calibrations were done using standard stars from the Landolt catalog (Landolt 1992) cov-

\footnotetext{
${ }^{4}$ http://terapix.iap.fr/soft/sextractor/index.html
}

ering a broad sample of magnitude and colors. A full description of the photometric procedure is beyond the scope of this work and will be discussed elsewhere (Le Fèvre et al., in preparation). In summary, we used the SA110 and SA101 star fields to measure the zero-points and color equations of each run. From these calibrations, we produced the magnitude histograms of each field in order to find out the cut off and a rough limiting magnitude. Although few fields have exposure time significantly larger than 1 hour, the depth of the sample is reasonably stable from field to field and reaches $I_{\mathrm{AB}}=24.5$ (this corresponds to a $5 \sigma$ detection within a 3 arcsec aperture). Up to this magnitude, 1.2 million galaxies were detected over the total area of $8.4 \mathrm{deg}^{2}$, and the final number density of galaxies over the usuable area of $6.5 \mathrm{deg}^{2}$ is $\sim 17$ galaxies $/ \operatorname{arcmin}^{2}$. This is about two times less than the number of detected galaxies because of the filtering processes described in the next section. 


\section{Shear measurements}

\subsection{Shape measurement}

The details of our shape measurement procedure and Point Spread Function (hereafter PSF) correction have been extensively described in two previous papers (Van Waerbeke et al. 2000; Maoli et al. 2001), and tested against numerical simulations (Erben et al. 2001). Therefore we will not reproduce these details here, but only give a short overview of the procedure. The shape measurement pipeline uses the IMCAT software (Kaiser et al. 1995$)^{5}$ combined with the SExtractor package. The different steps in the procedure are as follows:

- Object detection with Sextractor;

- The shape parameters defined in Kaiser et al. (1995) are calculated using IMCAT;

- Stars are identified in the stellar branch of the sizemagnitude diagram. Stars brighter than 1 magnitude below the saturation level are excluded. Objects smaller than the PSF size are discarded as galaxy candidates (because a shape correction below the PSF size is meaningless);

- The PSF is measured from the stars, and interpolated continuously over the CCD's using a third order polynomial;

- Galaxy shapes are corrected using the scheme developed in Kaiser et al. (1995), modified and adapted to our problem as described in Erben et al. (2001). The shape correction is a two-step process: first we remove the anisotropic contribution of the PSF, then the isotropic contribution is suppressed according to Luppino \& Kaiser (1997);

- A weight $w$ is calculated for each galaxy, which depends on the level of noise in the shape correction (see Eq. (7) in Van Waerbeke et al. 2000);

- For each galaxy pair with members closer than 15 pixels $(3$ arcsec), one member is removed, in order to avoid the problem of overlapping isophotes reported in Van Waerbeke et al. (2000);

- Each CCD is visualized by eye, and the bad areas are masked (star spikes and ghost images, blank lines or columns, fringe residuals). After the whole process of cleaning and object selection, 420000 galaxies were effectively used for the weak lensing analysis.

The raw ellipticity $e$ of a galaxy is measured from the second moments $I_{i j}$ of the surface brightness $f(\boldsymbol{\theta})$ :

$$
e=\left(\frac{I_{11}-I_{22}}{\operatorname{Tr}(I)} ; \frac{2 I_{12}}{\operatorname{Tr}(I)}\right), \quad I_{i j}=\int \mathrm{d}^{2} \theta W(\theta) \theta_{i} \theta_{j} f(\boldsymbol{\theta}) .(1)
$$

The window function $W(\boldsymbol{\theta})$ suppresses the noise at large distances from the object center. The procedure described above gives a corrected galaxy ellipticity $e^{\text {gal }}$ calculated

\footnotetext{
${ }^{5}$ Kindly made available by Nick Kaiser at http://www.ifa.hawaii.edu/ kaiser/
}
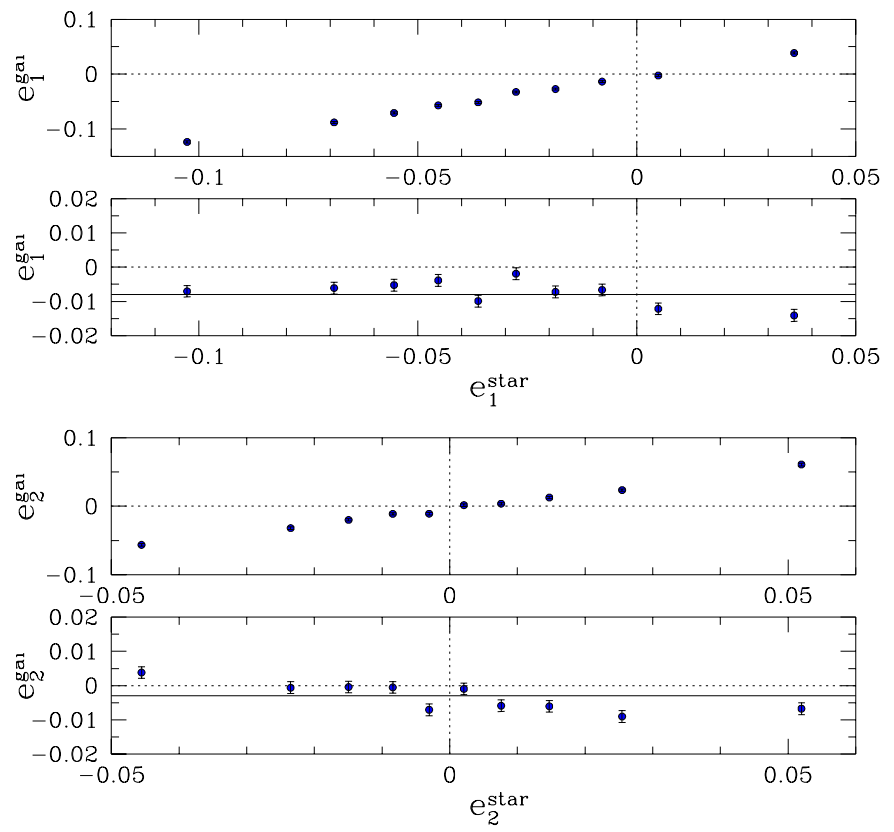

Fig. 1. Top and third panels: averaged $e_{1}^{\text {gal }}$ and $e_{2}^{\text {gal }}$ component versus the ellipticity of the PSF at the galaxy location, without the anisotropic PSF correction. Second from top and bottom panels: averaged $e_{1}^{\text {gal }}$ and $e_{2}^{\text {gal }}$ including the anisotropic PSF correction. A residual bias is the corrected ellipticity is present (shown by the two straight solid lines), as in our previous analysis, whose origin is still unclear. It is a constant bias which can be easily corrected for.

from the $e$ 's. According to Kaiser et al. (1995), the ensemble average of $e^{\text {gal }}$ is equal to the shear $\gamma$ at the galaxy location. Figure 1 shows the level of systematics in $e^{\text {gal }}$ with and without the anisotropic PSF correction. As quoted in Van Waerbeke et al. (2000), the galaxy ellipticities show a small offset $(-0.008,-0.003)$, whose origin is still unclear. However as it was demonstrated in Van Waerbeke et al. (2000), this systematic is independent of size and magnitude, and therefore it can be easily corrected for by shifting all ellipticities by the same amount. After correction, the average galaxy ellipticity is bounded between -0.005 and 0.005 , and the variance of the residual systematics is less than $\sim 10^{-5}$. It is worth mentioning that the aperture mass is not sensitive to this offset even if it were not corrected.

\section{Statistical measures of shear correlations}

\subsection{Theory}

We summarize the different statistics we shall measure, and how they depend on cosmological models. We concentrate on 2-point statistics and variances, since higher order moments are more difficult to measure, and will be addressed in a forthcoming paper.

Let us assume a normalised source redshift distribution parameterized as:

$n\left(z_{\mathrm{s}}\right)=\frac{\beta}{z_{0} \Gamma\left(\frac{1+\alpha}{\beta}\right)}\left(\frac{z_{\mathrm{s}}}{z_{0}}\right)^{\alpha} \exp \left[-\left(\frac{z_{\mathrm{s}}}{z_{0}}\right)^{\beta}\right]$, 


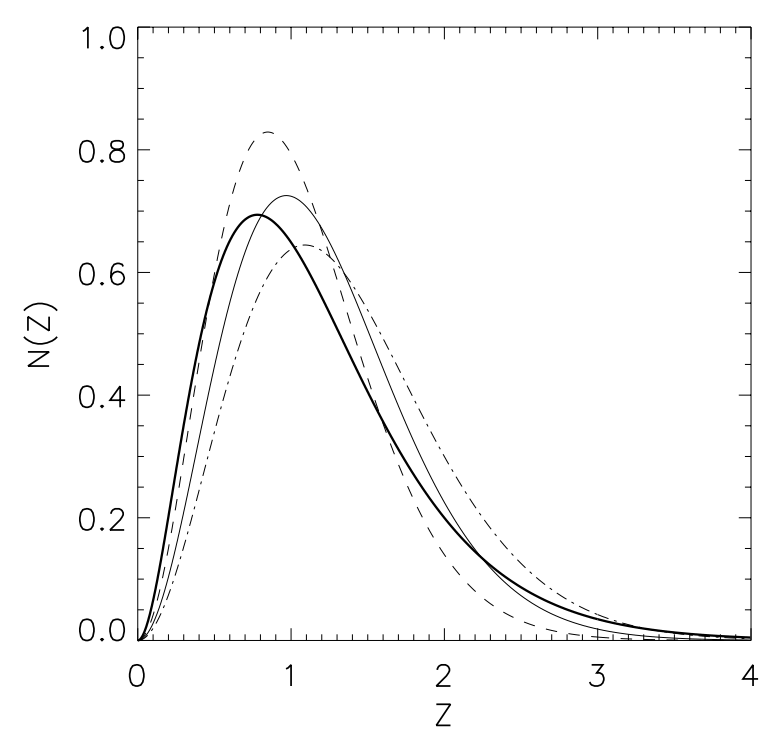

Fig. 2. The thin solid line shows our redshift distribution model given by Eq. (2) with $\left(z_{0}, \alpha, \beta\right)=(0.8,2,1.5)$. Two other models will also be used: one is $\left(z_{0}, \alpha, \beta\right)=(0.7,2,1.5)$ (thin dashed line) and one is $\left(z_{0}, \alpha, \beta\right)=(0.9,2,1.5)$ (thin dotdashed line). The thick solid line corresponds to the model used in Wilson et al. (2000) for the $I \simeq[22.5,23.5]$ galaxies. All the distributions are normalised.

with the parameters $\left(z_{0}, \alpha, \beta\right)=(0.8,2,1.5)$, which is consistent with a limiting magnitude $I_{\mathrm{AB}}=24.5$ given by Cohen et al. (2000) (it corresponds to a mean redshift of 1.2). However, in contrast to Cohen et al. (2000) we only have photometric data (in one color), which prevents us from inferring the accurate redshift distribution of our galaxies. The impact of this uncertainty is discussed below. We adopted a simplified approach consisting in looking at the sensitivity of cosmological parameter estimation for three realistic redshift distributions. Therefore in addition to the distribution expressed in Eq. (2) with $z_{0}=0.8$, we will consider two other sets, one is $\left(z_{0}, \alpha, \beta\right)=$ $(0.7,2,1.5)$ and the other $\left(z_{0}, \alpha, \beta\right)=(0.9,2,1.5)$, which $z_{0}=0.8 \pm 0.1$ (similar to the redshift error quoted in Rhodes et al. 2001), corresponding to an uncertainty in the mean redshift of \pm 0.15 . The three models are shown in Fig. 2, together with the redshift distribution used in Wilson et al. (2000) corresponding to a magnitude distribution $I \simeq[22.5,23.5]$, slightly brighter than our survey (the thick solid line).

We define the power spectrum of the convergence as (following the notation in Schneider et al. 1998):

$$
\begin{aligned}
P_{\kappa}(k)= & \frac{9}{4} \Omega_{0}^{2} \int_{0}^{w_{\mathrm{H}}} \frac{\mathrm{d} w}{a^{2}(w)} P_{3 \mathrm{D}}\left(\frac{k}{f_{\mathrm{K}}(w)} ; w\right) \\
& \times\left[\int_{w}^{w_{\mathrm{H}}} \mathrm{d} w^{\prime} n\left(w^{\prime}\right) \frac{f_{\mathrm{K}}\left(w^{\prime}-w\right)}{f_{\mathrm{K}}\left(w^{\prime}\right)}\right]^{2},
\end{aligned}
$$

where $f_{\mathrm{K}}(w)$ is the comoving angular diameter distance out to a distance $w$ ( $w_{\mathrm{H}}$ is the horizon distance), and $n(w(z))$ is the redshift distribution of the sources given in Eq. (2). $P_{3 \mathrm{D}}(k)$ is the non-linear mass power spectrum, and $k$ is the 2-dimensional wave vector perpendicular to the line-of-sight. For a top-hat smoothing window of radius $\theta_{c}$, the variance is:

$$
\left\langle\gamma^{2}\right\rangle=\frac{2}{\pi \theta_{\mathrm{c}}^{2}} \int_{0}^{\infty} \frac{\mathrm{d} k}{k} P_{\kappa}(k)\left[J_{1}\left(k \theta_{c}\right)\right]^{2},
$$

where $J_{1}$ is the first Bessel function of the first kind.

The aperture mass $M_{\text {ap }}$ was introduced in Kaiser et al. (1994):

$M_{\mathrm{ap}}=\int_{\theta<\theta_{c}} \mathrm{~d}^{2} \boldsymbol{\theta} \kappa(\boldsymbol{\theta}) U(\theta)$,

where $\kappa(\boldsymbol{\theta})$ is the convergence field, and $U(\theta)$ is a compensated filter (i.e. with zero mean). Schneider et al. (1998) applied this statistic to the cosmic shear measurements. They showed that the aperture mass variance is related to the convergence power spectrum by:

$\left\langle M_{\mathrm{ap}}^{2}\right\rangle=\frac{288}{\pi \theta_{c}^{4}} \int_{0}^{\infty} \frac{\mathrm{d} k}{k^{3}} P_{\kappa}(k)\left[J_{4}\left(k \theta_{c}\right)\right]^{2}$.

$\left\langle M_{\mathrm{ap}}^{2}\right\rangle$ can be calculated directly from the shear $\gamma$ without the need for a mass reconstruction.

For each galaxy, we define the tangential and radial shear components $\left(\gamma_{\mathrm{t}}\right.$ and $\left.\gamma_{\mathrm{r}}\right)$ with respect to the center of the aperture:

$$
\begin{aligned}
& \gamma_{t}=-\gamma_{1} \cos (2 \phi)-\gamma_{2} \sin (2 \phi) \\
& \gamma_{r}=-\gamma_{2} \cos (2 \phi)+\gamma_{1} \sin (2 \phi),
\end{aligned}
$$

where $\phi$ is the position angle between the $x$-axis and the line connecting the aperture center to the galaxy. It is then easy to show that the aperture mass is related to the tangential shear by:

$$
M_{\mathrm{ap}}=\int_{\theta<\theta_{c}} \mathrm{~d}^{2} \boldsymbol{\theta} \gamma_{\mathrm{t}}(\boldsymbol{\theta}) Q(\theta)
$$

where the filter $Q(\theta)$ is given from $U(\theta)$ :

$Q(\theta)=\frac{2}{\theta^{2}} \int_{0}^{\theta} \mathrm{d} \theta^{\prime} \theta^{\prime} U\left(\theta^{\prime}\right)-U(\theta)$

If $\gamma_{\mathrm{t}}$ is replaced by $\gamma_{\mathrm{r}}$ in Eq. (8), then the lensing signal vanishes, due to the curl-free property of the shear field $\left(\right.$ Kaiser et al. 1994) ${ }^{6}$. This remarkable property constitutes a test of the lensing origin of the signal. The change from $\gamma_{\mathrm{t}}$ to $\gamma_{\mathrm{r}}$ can simply be accomplished just by rotating the galaxies by 45 degrees in the aperture (i.e. changing a curlfree field to a pure curl field). Hereafter we call the $M_{\text {ap }}$ statistic measured with the 45 degree rotated galaxies the $R$-mode ( $R$ for radial mode), and $\left\langle M_{\perp}^{2}\right\rangle$ the corresponding variance. It is interesting to note that the $R$-mode is not expected to vanish if the measured signal is due to spin alignments of galaxies (Crittenden et al. 2000b). Therefore it can be used to constrain the amount of residual systematics as well as the degree of the spin alignment of the galaxies leading to their intrinsic alignment.

\footnotetext{
${ }^{6}$ Curl modes are produced by non-linear lensing effects, but these are very small (Bernardeau et al. 1997).
} 
From the shear $\gamma$ and its projections defined in Eq. (7) we can also define various galaxy pairwise correlation functions related to the convergence power spectrum. Note that the tangential and radial shear projections in what follows are performed using the relative location vector of the pair members, not from an aperture center. The following correlation functions can be defined (MiraldaEscudé 1991; Kaiser 1992):

$\langle\gamma \gamma\rangle_{\theta}=\frac{1}{2 \pi} \int_{0}^{\infty} \mathrm{d} k k P_{\kappa}(k) J_{0}(k \theta)$

$\left\langle\gamma_{\mathrm{t}} \gamma_{\mathrm{t}}\right\rangle_{\theta}=\frac{1}{4 \pi} \int_{0}^{\infty} \mathrm{d} k k P_{\kappa}(k)\left[J_{0}(k \theta)+J_{4}(k \theta)\right]$

$\left\langle\gamma_{\mathrm{r}} \gamma_{\mathrm{r}}\right\rangle_{\theta}=\frac{1}{4 \pi} \int_{0}^{\infty} \mathrm{d} k k P_{\kappa}(k)\left[J_{0}(k \theta)-J_{4}(k \theta)\right]$

where $\theta$ is the pair separation angle. The cross-correlation $\left\langle\gamma_{\mathrm{t}} \gamma_{\mathrm{r}}\right\rangle_{\theta}$ is expected to vanish for parity reasons (there is no preferred orientation on average).

It is easy to see that the Eqs. (4), (6), (10)-(12) are different ways to measure the same quantity, that is the convergence power spectrum $P_{\kappa}(k)$. Ultimately the goal is to deproject $P_{\kappa}(k)$ in order to reconstruct the 3D mass power spectrum from Eq. (3), but this is beyond the scope of this paper. Here we restrict our analysis to a joint detection of these statistics, and show that they are consistent with the gravitational lensing hypothesis. We will also examine the constraints on the power spectrum normalization $\sigma_{8}$ and the mean density of the universe $\Omega_{0}$.

\subsection{Estimators}

Let us now define the estimators we used to measure the quantities given in Eqs. (4), (6), (10)-(12).

The variance of the shear is simply obtained by a cell averaging of the squared shear $\gamma^{2}\left(\boldsymbol{\theta}_{i}\right)$ over the cell index $i$. An unbiased estimate of the squared shear for the cell $i$ is:

$E\left[\gamma^{2}\left(\boldsymbol{\theta}_{i}\right)\right]=\frac{\sum_{\alpha=1}^{2} \sum_{k \neq l}^{N_{i}} w_{k} w_{l} e_{\alpha}^{\mathrm{gal}}\left(\boldsymbol{\theta}_{k}\right) e_{\alpha}^{\mathrm{gal}}\left(\boldsymbol{\theta}_{l}\right)}{\sum_{k \neq l}^{N_{i}} w_{k} w_{l}}$,

where $w_{k}$ is the weight for the galaxy $k$, and $N_{i}$ is the number of galaxies in the cell $i$. The cell averaging over the survey is then an unbiased estimate of the shear variance $\left\langle\gamma^{2}\right\rangle$. However, due to the presence of masked areas (mentioned in Sect. 4.1), some cells may have a very low number of galaxies compared to others. Instead of applying an arbitrary sharp cut off on the fraction of the apertures filled with masks (as in previous works) we decided to keep all the cells, and to weight each of them with the squared sum of the galaxy weights located in the cell. The cell averaging is now defined as:

$E\left[\gamma^{2}\right]=\frac{\sum_{\text {cells }}\left[E\left[\gamma^{2}\left(\boldsymbol{\theta}_{i}\right)\right]\left(\sum_{k=1}^{N_{i}} w_{k}\right)^{2}\right]}{\sum_{\text {cells }}\left[\left(\sum_{k=1}^{N_{i}} w_{k}\right)^{2}\right]}$,

where $i$ identifies the cell. One potential problem with this procedure is that the sum of the weights is related to the number of objects in the aperture, which is affected by magnification bias, and therefore correlated with the shear signal measured in the same aperture. Fortunately the first non-vanishing contribution of this weighting scheme is a third order effect (of order 1\%), and is therefore negligi$\mathrm{ble}^{7}$. The advantage is that we can use all cells without wondering about their filling factor, and it naturaly downweights the cells which contain a large fraction of poorly determined galaxy ellipticities. The weighting scheme of Eq. (14) has been tested against numerical simulation, using a simulated survey with the same survey geometry as our data: it gave unbiased measures of the lensing signal applied to the galaxies.

The $M_{\text {ap }}$ statistic is calculated from a similar estimator, although the smoothing window is no longer a top-hat but the $Q$ function defined in Eq. (9). An unbiased estimate of $M_{\mathrm{ap}}^{2}\left(\boldsymbol{\theta}_{i}\right)$ in the cell $i$ is:

$E\left[M_{\mathrm{ap}}^{2}\left(\boldsymbol{\theta}_{i}\right)\right]=\frac{\sum_{k \neq l}^{N_{i}} w_{k} w_{l} e_{\mathrm{t}}^{\mathrm{gal}}\left(\boldsymbol{\theta}_{k}\right) e_{\mathrm{t}}^{\mathrm{gal}}\left(\boldsymbol{\theta}_{l}\right) Q\left(\theta_{k}\right) Q\left(\theta_{l}\right)}{\sum_{k \neq l}^{N} w_{k} w_{l}}$,

where $e_{\mathrm{t}}^{\text {gal }}$ is the tangential galaxy ellipticity, and $Q$ is given by (see Schneider et al. 1998):

$Q(\theta)=\frac{6}{\pi}\left(\frac{\theta}{\theta_{c}}\right)^{2}\left[1-\left(\frac{\theta}{\theta_{c}}\right)^{2}\right]$.

The estimation of $\left\langle M_{\mathrm{ap}}^{2}\right\rangle$ over the survey is then given by the same expression as in Eq. (14), with $E\left[\gamma^{2}\left(\boldsymbol{\theta}_{i}\right)\right]$ replaced by $E\left[M_{\text {ap }}^{2}\left(\boldsymbol{\theta}_{i}\right)\right]$. We emphasize that the this filter probes effective scales $\theta_{\mathrm{c}} / 5$, and not $\theta_{\mathrm{c}}$ (see Fig. 2 in Schneider et al. 1998). Therefore we have to be careful when comparing the signal at different scales between different estimators.

The shear correlation function $\langle\gamma \gamma\rangle_{\theta}$ at separation $\theta$ is obtained by identifying all the pairs of galaxies falling in

\footnotetext{
${ }^{7}$ Moreover the slope of number counts in our $I$-band is $\sim 0.3$, which makes the magnification effect very small (see Moessner et al. 1998 for an application of the effect to the angular correlation function).
} 


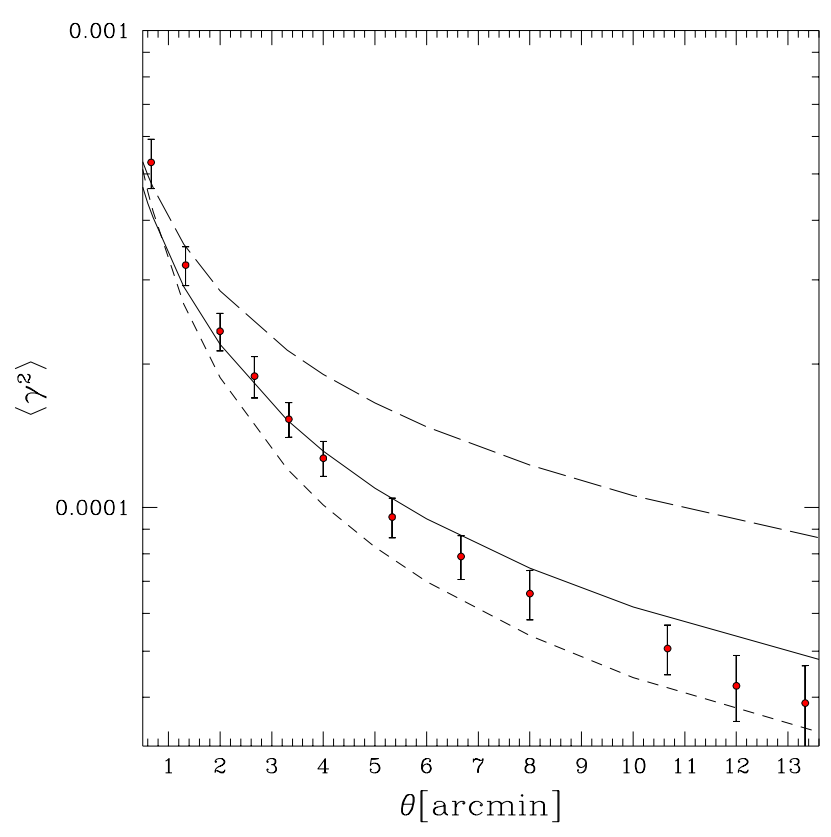

Fig. 3. Top-hat smoothed variance of the shear (points with error bars). The three models correspond to $\left(\Omega_{0}, \Lambda, \sigma_{8}\right)=$ $(0.3,0,0.9),(0.3,0.7,0.9),(1,0,0.6)$ for the short-dashed, solid and long-dashed lines respectively. The power spectrum is a CDM-model with $\Gamma=0.21$. The error bars correspond to the dispersion of the variance measured from 200 realizations of the data set with randomized orientations of the galaxy ellipticities.

the separation interval $[\theta-\mathrm{d} \theta, \theta+\mathrm{d} \theta]$, and calculating the pairwise shear correlation:

$E[\gamma \gamma ; \theta]=\sum_{\alpha=1}^{2} \frac{\sum_{\text {pairs }} w_{k} w_{l} e_{\alpha}^{\text {gal }}\left(\boldsymbol{\theta}_{k}\right) e_{\alpha}^{\text {gal }}\left(\boldsymbol{\theta}_{l}\right)}{\sum_{\text {pairs }} w_{k} w_{l}}$.

The tangential and radial correlation functions $\left\langle\gamma_{\mathrm{t}} \gamma_{\mathrm{t}}\right\rangle_{\theta}$ and $\left\langle\gamma_{\mathrm{r}} \gamma_{\mathrm{r}}\right\rangle_{\theta}$ are measured also from Eq. (17) by replacing $e^{\text {gal }}$ with $e_{\mathrm{t}}^{\text {gal }}$ and $e_{\mathrm{r}}^{\text {gal }}$ respectively and dropping the sum over $\alpha$. It is worth noting that the estimators given here are independent of the angular correlation properties of the source galaxies.

\section{Results and comparison to cosmological models}

In this section we present our measurements of the 2-point correlations of the shear using the different estimators defined above. Figures 3 to 8 show the results for the different estimators: the variance in Fig. 3, the mass aperture statistic in Fig. 4, the shear correlation function in Fig. 5, the radial and tangential shear correlations in Fig. 6, and the cross-correlation of the radial and tangential shear in Fig. 8. Along with the measurements we show the predictions of three cosmological models which are representative of an open model, a flat model with cosmological constant, and an Einstein-de Sitter model. The amplitude of mass fluctuations in these models is normalized to the abundance of galaxy clusters. The three models are char-

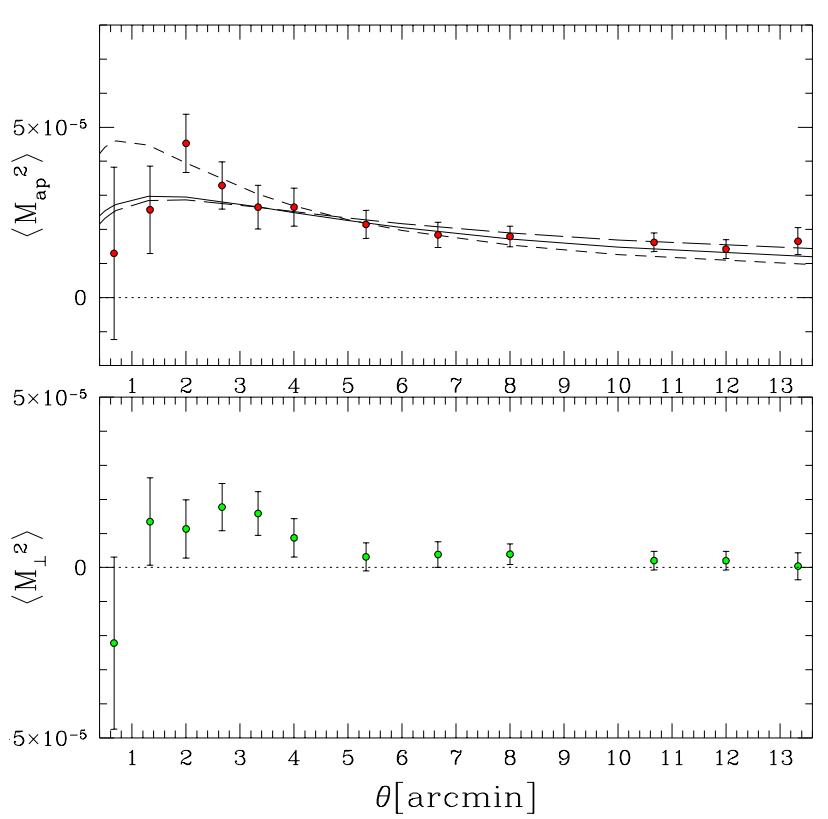

Fig. 4. The aperture mass statistic for the same models as in Fig. 3 . The lower panel plots the $R$-mode, obtained by making a 45 degree rotation as described in the text. There is no significant detection for $\theta>5$ arcmin (corresponding to an effective angular scale of $1^{\prime}$, as discussed in the text), which shows the low level of contamination by galaxy intrinsic alignment and/or residual systematics.

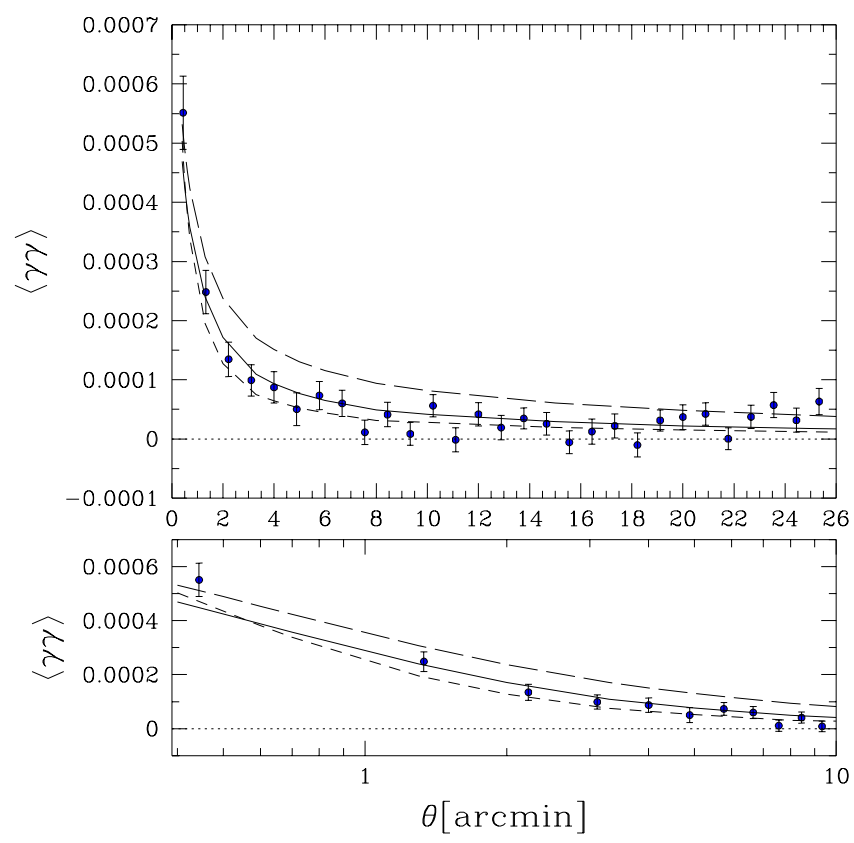

Fig. 5. Shear correlation function $\langle\gamma \gamma\rangle_{\theta}$. The models are the same as in Fig. 3. The lower panel uses a log-scale for the $x$-axis to highlight the small scale details.

acterized by the values of $\Omega_{0}, \Lambda$ and $\sigma_{8}$ as follows:

- short-dashed line: $\Omega_{0}=0.3, \Lambda=0, \sigma_{8}=0.9$

- solid line: $\Omega_{0}=0.3, \Lambda=0.7, \sigma_{8}=0.9$

- long-dashed line: $\Omega_{0}=1, \Lambda=0, \sigma_{8}=0.6$.

The power spectrum is taken to be a cold dark matter (CDM) power spectrum with shape parameter $\Gamma=0.21$. 


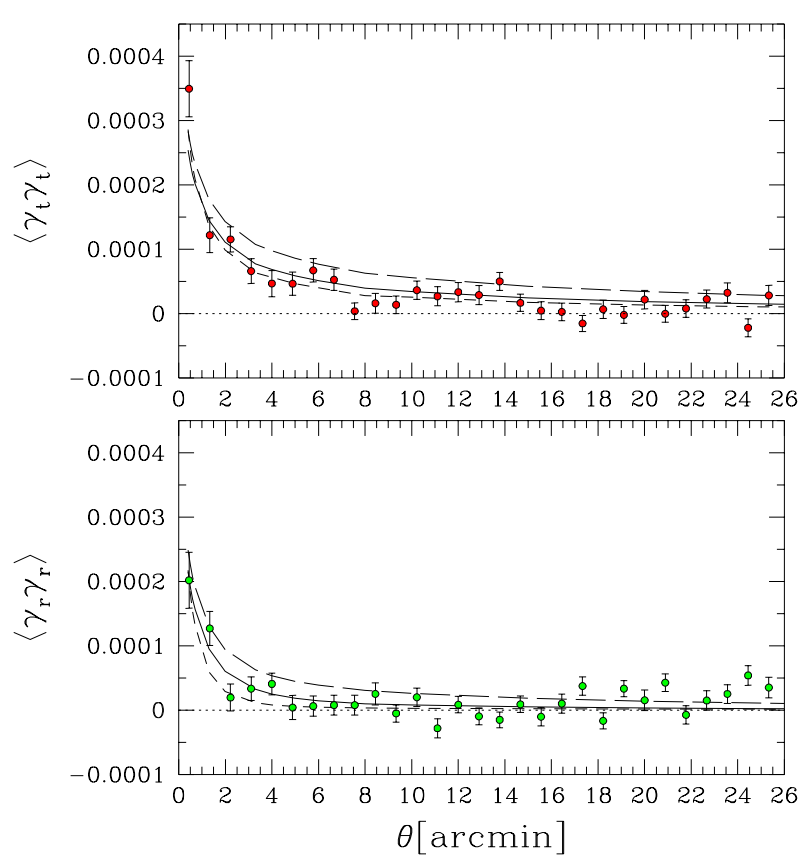

Fig. 6. Top panel: tangential shear correlation function $\left\langle\gamma_{t} \gamma_{t}\right\rangle_{\theta}$. Bottom panel: radial shear correlation function $\left\langle\gamma_{\mathrm{r}} \gamma_{\mathrm{r}}\right\rangle_{\theta}$. The models are the same as in Fig. 3.

The predictions for shear correlations are computed using the non-linear evolution of the power spectrum using the Peacock \& Dodds (1996) fitting formula. It is assumed that the source redshift distribution follows Eq. (2) with $\left(z_{0}, \alpha, \beta\right)=(0.8,2,1.5)$, which corresponds to a mean redshift of 1.2 .

It is reassuring that the different statistics agree with each other in their comparison with the model predictions. These statistics weight the data in different ways and are susceptible to different kinds of systematic errors. The consistency of all the 2-point estimators suggests that the level of systematics in the data is low compared to the signal. A further test for systematics is provided by measuring the cross-correlation function $\left\langle\gamma_{\mathrm{t}} \gamma_{\mathrm{r}}\right\rangle_{\theta}$, which should be zero for a signal due to gravitational lensing. It is shown in Fig. 8 that it is indeed consistent with zero at all scales. The figure also shows the cross-correlation obtained when the anisotropic contamination of the PSF is not corrected - clearly such a correction is crucial in measuring the lensing signal.

The lower panel of Fig. 4 shows the $R$-mode of the mass aperture statistic. As this statistic uses a compensated filter, the scale beyond which the measured $R$-mode is consistent with zero ( $5^{\prime}$ on the plot) corresponds to an effective angular scale $\theta \simeq 1^{\prime}$. This places an upper limit on measured shear correlations due to the intrinsic alignment of galaxies, given the redshift distribution of the sources. The vanishing of $\left\langle M_{\perp}^{2}\right\rangle$ for effective angular scales larger than $1^{\prime}$ strongly supports our conclusion that the level of residual systematics is low: this is a very hard test to pass, as it means that the signal is produced by a pure scalar field, which need not be the case for systematics. We checked that $M_{\perp}^{2}$ is Gaussian distributed with a zero

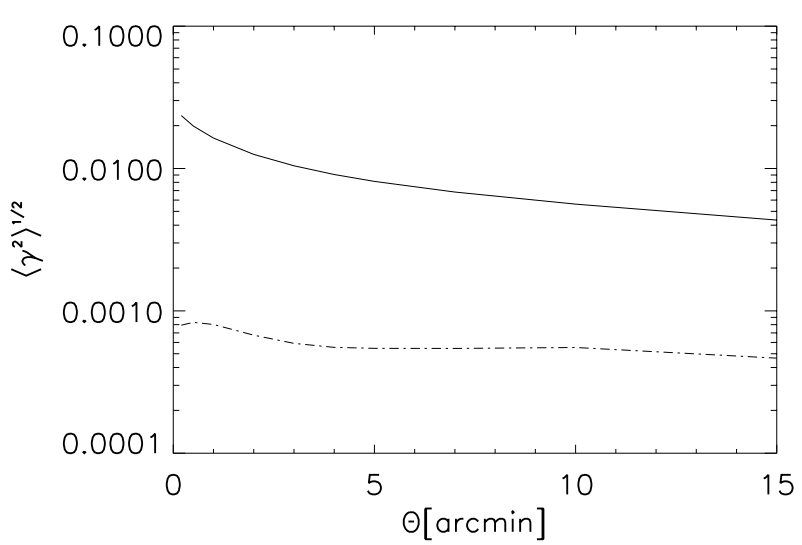

Fig. 7. Shear rms $\left\langle\gamma^{2}\right\rangle^{1 / 2}$ (solid line) measured in a ray-tracing simulation (Jain et al. 2000) for the open $\Omega_{0}=0.3$ model. The dashed line is the sample variance of the shear rms measured from 7 different realisations of the mass distribution for a survey of $6.5 \mathrm{deg}^{2}$.

average all over the survey, which is what we would expect from a pure noise realisation. For scales below $5^{\prime}$ on the plot, the $R$-mode is not consistent with zero at the $2-\sigma$ level. Since the cross-correlation $\left\langle\gamma_{t} \gamma_{\mathrm{r}}\right\rangle_{\theta}$ is consistent with zero at this scale, the source of the $R$-mode is probably not a residual systematic caused by an imperfect PSF correction. Rather, it might be due to the effect of intrinsic alignments (Crittenden et al. 2000b).

The error bars shown in Figs. 3 to 8 are calculated from a measurement of the different statistics in 200 realizations of the data set, with randomized orientations of the galaxies. We measured the sample variance from raytracing simulations (Jain et al. 2000) and find that it is smaller than $20 \%$ of the noise error bars shown here (see Van Waerbeke et al. 1999 where the sample variance has been calculated for surveys with similar geometry), therefore we have not included it in our figures. Figure 7 shows an estimate of the sample variance for the rms shear using a compact $6.5 \mathrm{deg}^{2}$ ray-tracing simulation (Jain et al. 2000). This figure shows that the sample variance is about one order of magnitude smaller than $\left\langle\kappa^{2}\right\rangle^{1 / 2}$ for the range of scales of interest. Hence our errors are not dominated by sample variance, as was the case in the first detections of cosmic shear. As the probed angular scales approach the size of the fields (which is $\sim 30^{\prime}$ with the CFH12K camera) the sample variance becomes larger. This could be responsible for the small fluctuations in the measured correlations in Figs. 5 and 6 for scales larger than $24^{\prime}$.

\section{Cosmological constraints}

As noted elsewhere (e.g. Bernardeau et al. 1997; Jain \& Seljak 1997), the parameters which dominate the 2-point shear statistics are the power spectrum normalization $\sigma_{8}$ and the mean density $\Omega_{0}$. We investigate below how the statistics measured in Figs. 3 to 6 are consistent with each other when constraining these parameters. Our parameter estimates below rely on some simplifying assumptions; a more detailed analysis over a wider space of parameters will be presented elsewhere. In particular, as discussed later, the choice of the slope of the power spectrum $\Gamma$ is 


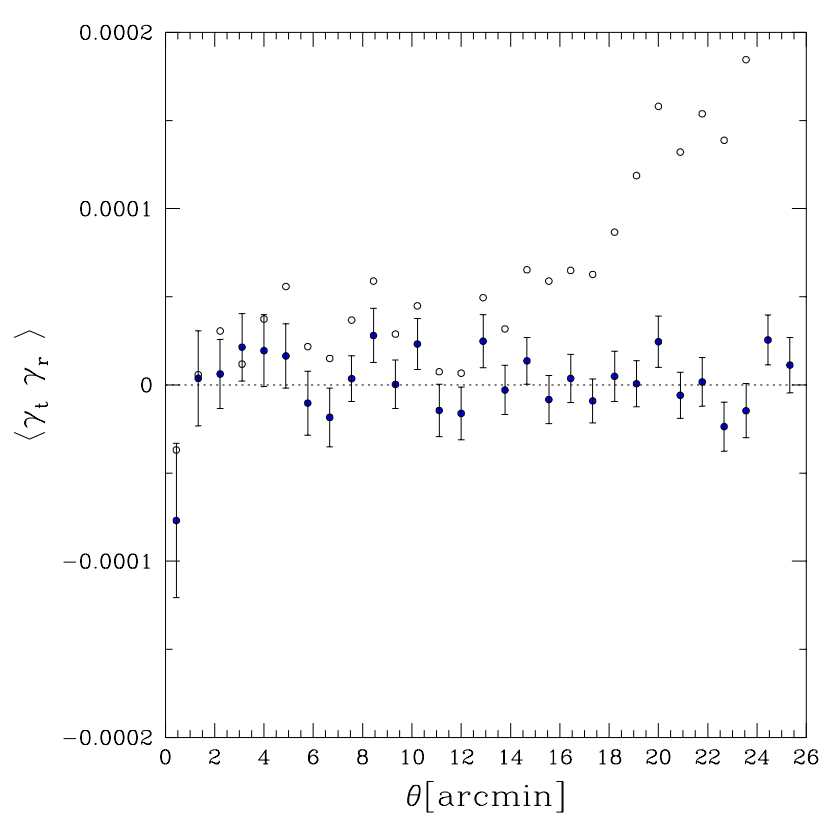

Fig. 8. Shear cross-correlation function $\left\langle\gamma_{\mathrm{t}} \gamma_{\mathrm{r}}\right\rangle_{\theta}$. The signal should vanish if the data are not contaminated by systematics. As a comparison, the open circles show the same crosscorrelation function computed from the galaxy ellipticities where the anisotropic correction of the PSF has been skipped.

weakly known, and may significantly affect the parameter estimate. As pointed out in Sect. 4, the uncertainty on the redshift distribution is also a concern, but we partialy address this point by constraining the parameters using three different redshift distributions. A complete analysis involving marginalisation over $\Gamma$ and the redshift distribution using tight priors is left for a future work.

We assume that the data follow Gaussian statistics and neglect sample variance since it is a very small contributor to the noise for our survey, as discussed above. We compute the likelihood function $\mathcal{L}$ :

$$
\mathcal{L}=\frac{1}{(2 \pi)^{n / 2}|\boldsymbol{S}|^{1 / 2}} \exp \left[-\frac{1}{2}(\boldsymbol{d}-\boldsymbol{s})^{T} \boldsymbol{S}^{-1}(\boldsymbol{d}-\boldsymbol{s})\right]
$$

where $\boldsymbol{d}$ and $\boldsymbol{s}$ are the data and model vectors respectively, and $\boldsymbol{S}=\left\langle(\boldsymbol{d}-\boldsymbol{s})^{T}(\boldsymbol{d}-\boldsymbol{s})\right\rangle$ is the noise correlation matrix. $\boldsymbol{S}$ was computed for the different statistics from 200 random realizations of the survey, therefore effects associated with the survey geometry are included in our noise matrix. The model $s$ was computed for a grid of cosmological models which covers $\Omega_{0} \in[0,1]$ and $\sigma_{8} \in[0.2,1.8]$ with a zero cosmological constant. The prior is chosen to be flat over this grid, and zero outside. We also fixed $\Gamma=0.21$ and we first use the redshift distribution given by Eq. (2) with $\left(z_{0}, \alpha, \beta\right)=(0.8,2,1.5)$. The two other redshift distributions defined in Sect. 4.1 will be discussed at the end of this section. We discuss below the impact of this choice of priors.

Figure 4 (bottom panel) shows that for effective scales smaller than $1^{\prime}$ there is a non-vanishing $R$-mode which could come either from a residual systematic, or from an intrinsic alignment effect. Therefore it is safer to exclude

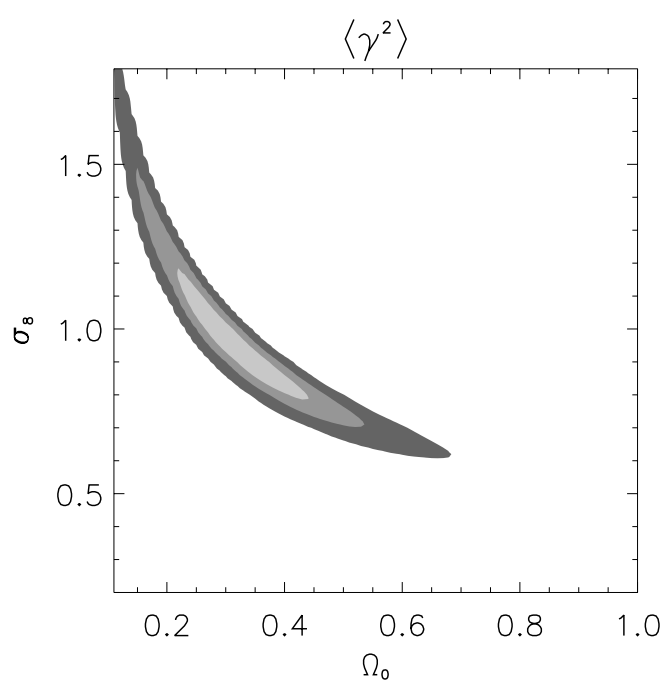

Fig. 9. Likelihood contours in the $\Omega_{0}-\sigma_{8}$ plane from the tophat smoothed variance $\left\langle\gamma^{2}\right\rangle$ shown in Fig. 3. The first point in Fig. 3 was not included in the likelihood calculation to avoid the small scale systematic shown in Fig. 4 (bottom panel). The cosmological models have $\Lambda=0$, with a CDM-type power spectrum and $\Gamma=0.21$. The redshift of the sources is given by Eq. (2). with $\left(z_{0}, \alpha, \beta\right)=(0.8,2,1.5)$. The confidence levels are $(0.68,0.95,0.999)$.

this part from the likelihood calculation: for the top-hat variance, we excluded the point at $1^{\prime}$, for the correlation functions the points below $2^{\prime}$, and for the $M_{\text {ap }}$ statistic the points below $5^{\prime}$. For the correlation function, we also excluded the points at scales larger than $20^{\prime}$ because of the small fluctuations in the measured correlations. The constraints on the cosmological parameters are not significantly affected whether these large scale points are excluded or not.

Figures 9 to 13 show the $\left(\Omega_{0}, \sigma_{8}\right)$ constraints for each of the statistics shown in Figs. 3 to 6 . The contours show the $99.9 \%, 95.0 \%$ and $68.0 \%$ confidence levels. The agreement between the contours is excellent, though the $M_{\text {ap }}$ statistic and the radial correlation function do not give as tight constraints as the other statistics. The correlation function measurements below $2^{\prime}$ may be considered by using error bars that include a possible systematic bias: this is equivalent to adding a systematic covariance matrix $\boldsymbol{S}^{\text {sys }}$ to the noise covariance $\boldsymbol{S}$ matrix in Eq. (18). The new contours computed with the enlarged error bars ${ }^{8}$ are shown in Fig. 14. The maximum of the likelihood in the variance and correlation function likelihood plots is at $\sigma_{8} \simeq 0.9$ and $\Omega_{0} \simeq 0.3$. Note that the results are in very good agreement ${ }^{9}$ with a similar plot in Maoli et al. (2001) (Fig. 8), here the contours are narrower, and are obtained from a homogeneous data set. Moreover, the degeneracy between $\Omega_{0}$ and $\sigma_{8}$ is broken.

\footnotetext{
8 The enlarged error bars were computed from the estimation of our $B$-mode analysis, which will be presented elsewhere.

9 although in Maoli et al. (2001) we performed a fit in the $\left(\Omega_{0}, \sigma_{8}\right)$ plane while here we measured the allowed area for the product $\sigma_{8} \Omega_{0}^{0.6}$, i.e. fixing the $\Omega_{0}$ exponent to 0.6 .
} 


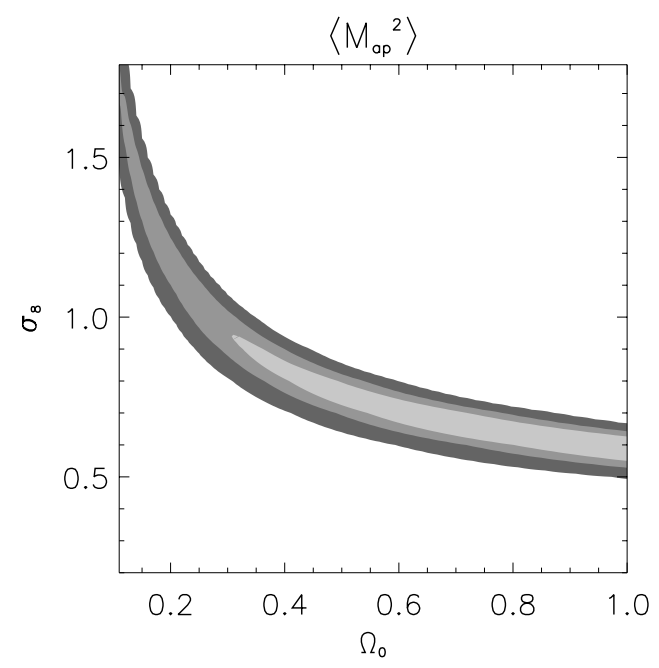

Fig. 10. As in Fig. 9, but using the $M_{\text {ap }}$ statistic of Fig. 4 (top panel) instead of the top-hat variance. The first five points in Fig. 4 were not included in the likelihood calculation in order to avoid the small scale systematic shown in Fig. 4 (bottom panel).

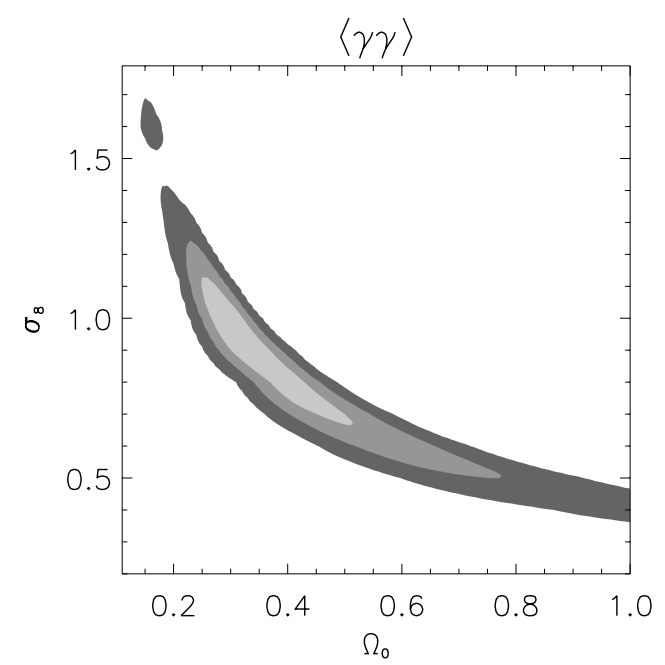

Fig. 11. Likelihood contours as in Fig. 9, but using the shear correlation function $\langle\gamma \gamma\rangle_{\theta}$ (Fig. 5) instead of the top-hat variance. The first two points and scales larger than $20^{\prime}$ in Fig. 5 were not included in the likelihood calculation to avoid the contribution from the small scale systematic shown in Fig. 4 (bottom panel).

The partial breaking of degeneracy between $\Omega_{0}$ and $\sigma_{8}$ was expected from the fully non-linear calculation of shear correlations (Jain \& Seljak 1997). In the non-linear regime the dependence of the 2-points statistics on $\Omega_{0}$ and $\sigma_{8}$ becomes sensitive to angular scale. For example, as shown in Jain \& Seljak (1997), the shear rms measures $\sigma_{8} \Omega_{0}^{0.5}$ on scale between $2^{\prime}-5^{\prime}$, and $\sigma_{8} \Omega_{0}^{0.8}$ on scales $\gtrsim 10^{\prime}$. Therefore a low $\Omega_{0}$ universe should see a net decrease of shear power at large scale compared to a $\Omega_{0}=1$ universe (for a given shape of the power spectrum), as is evident in Fig. 3. Note that the aperture mass $M_{\text {ap }}$ is still degenerate with $\Omega_{0}$ and $\sigma_{8}$ (Fig. 10) because it probes effective scales up to $\sim 2.6^{\prime}$ only, which is not enough to break the degeneracy.

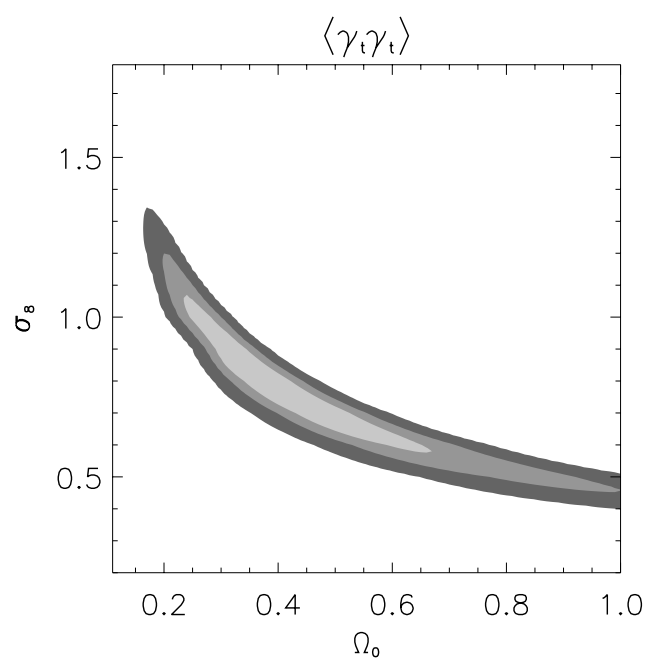

Fig. 12. As in Fig. 9, but using the tangential shear correlation function $\left\langle\gamma_{t} \gamma_{t}\right\rangle_{\theta}$ (Fig. 6) instead of the top-hat variance. The first two points and scales larger than $20^{\prime}$ in Fig. 6 were not included in the likelihood calculation in order to avoid the contribution from the small scale systematic shown in Fig. 4 (bottom panel).

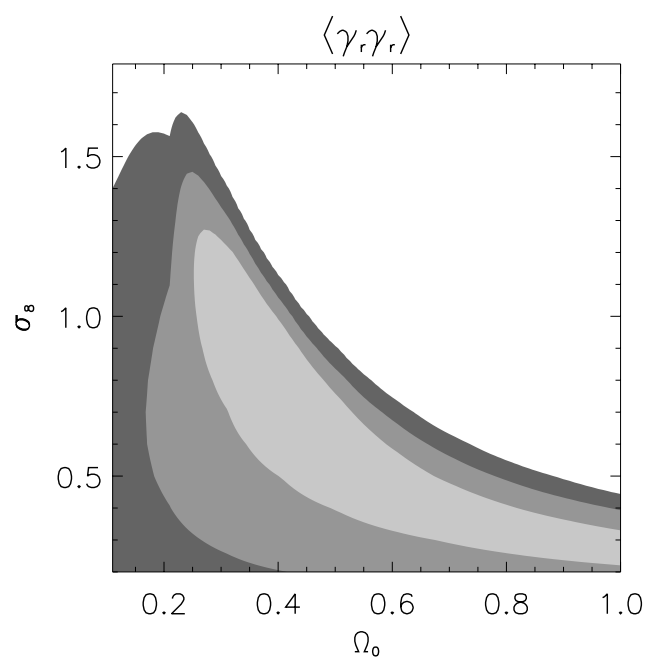

Fig. 13. As in Fig. 9, but using the radial shear correlation function $\left\langle\gamma_{\mathrm{r}} \gamma_{\mathrm{r}}\right\rangle_{\theta}$ (results in Fig. 6) instead of the top-hat variance. The first two points and scales larger than $20^{\prime}$ in Fig. 6 were not included in the likelihood calculation in order to avoid the contribution from the small scale systematic shown in Fig. 4 (bottom panel).

It seems that the aperture mass (Fig. 10) gives a slightly larger $\sigma_{8}$ for a large $\Omega_{0}$ compared to the other statistics, while they all agree for $\Omega_{0}<0.7$. This could be an indication for a low $\Omega_{0}$ Universe, however in practice, the probability contours for the different statistics cannot be combined in a straightforward way because they are largely redundant. The best strategy here is to concentrate on one particular statistic. We expect the best constraints from the shear correlation function (since it contains all the information by definition), and therefore base our parameter estimates on the likelihood contours obtained from it. The contours in the $\sigma_{8}-\Omega_{0}$ plane in Fig. 14 closely follow the curve $\sigma_{8} \propto \Omega_{0}^{0.6}$. This allows us 


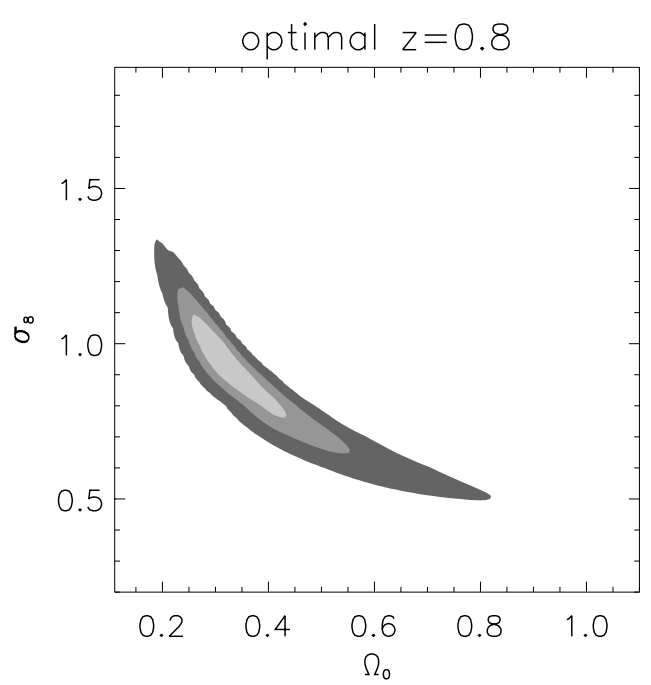

Fig. 14. Likelihood contours as in Fig. 11, but all the points in Fig. 5 on scales smaller than $20^{\prime}$ were used. In order to account for the small scale systematic shown in Fig. 4 (bottom panel) the error bars on the first two points were increased to include the systematic amplitude.

to obtain the following measurement of $\sigma_{8} \Omega_{0}^{0.6}$ (from this figure alone):

$\sigma_{8} \Omega_{0}^{0.6}=0.48_{-0.05(0.07)}^{+0.04(0.06)}$

where the uncertainties correspond to the $95 \%(99.9 \%)$ confidence levels. The result in Eq. (19) is derived for CDM models only, and fairly robust against different values of $\Gamma$. These errors are statistical only, and that they do not include systematic error on the redshift distribution and on the value of $\Gamma$.

If we choose a strong prior for $\Gamma$, we can constrain the two parameters separately; for $\Gamma=0.21$ we get, at the $95 \%$ confidence level: $0.22<\Omega_{0}<0.55$ and $0.65<\sigma_{8}<1.2$ for open models and $\sigma_{8}>0.7$ and $\Omega_{0}<0.4$ for flat $(\Lambda$ CDM) models. However, this result is clearly sensitive to the prior choosen for $\Gamma$. In particular, if we use the relation $\Gamma=\Omega_{0} h$ for a cold dark matter model, then some extreme combinations of $\sigma_{8}, \Omega_{0}$ and $\Gamma$ cannot be ruled out from lensing alone. The degeneracy between $\Omega_{0}$ and $\sigma_{8}$ is broken only if we take $\Gamma$ to lie in a reasonable interval. Such interval can be motivated by galaxy surveys for instance, which give $0.19<\Gamma<0.37$ at $68 \%$ confidence level for the APM (Eisentsein \& Zaldarriaga 2001). For instance the choice $\Gamma=0.7$ would make $\Omega_{0}=1, \sigma_{8}=0.5$ consistent with the data. The second source of uncertainty comes from the redshift distribution, kown only approximately. As discussed in Sect. 4.1 and shown in Fig. 2 we have a rough idea of this distribution, but until we obtain the information on the photometric or spectroscopic redshifts (which is in progress) we cannot guarantee a precise cosmological parameter estimation here. Figures 15 and 16 show the confidence contours as calculated in Fig. 14 but with the two other redshift distributions defined in Sect. 4.1. Despite the large differences of the distribution, in particular for the number of galaxies at $z>1.5$, it is reassuring that the contours are in fact

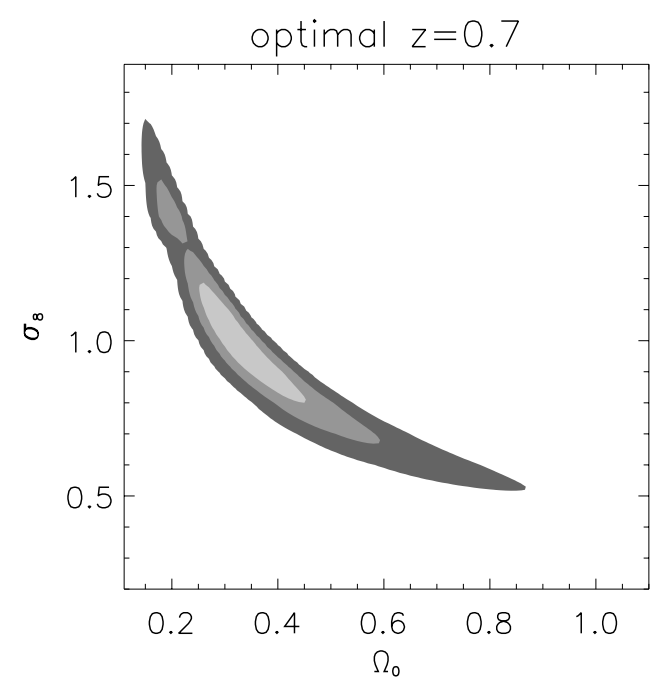

Fig. 15. Likelihood contours as in Fig. 14, but the source redshift distribution is assumed to be lower, with $\left(z_{0}, \alpha, \beta\right)=$ $(0.7,2,1.5)$.

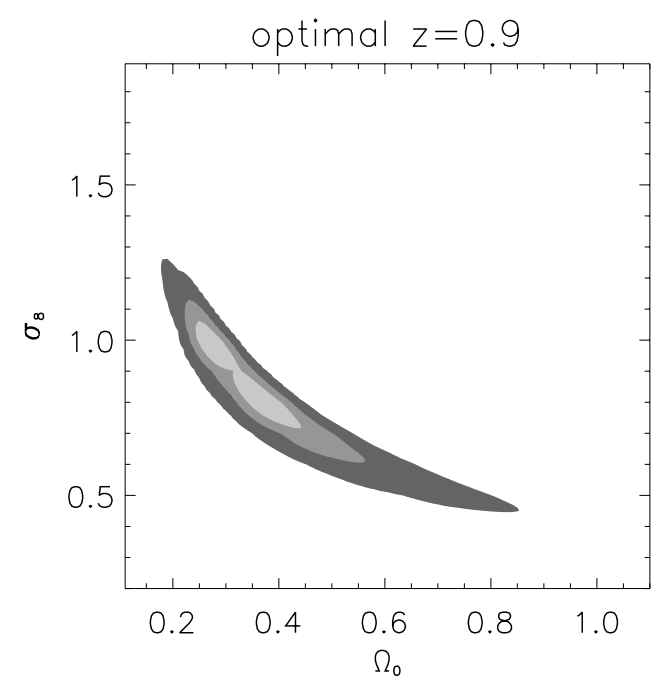

Fig. 16. Likelihood contours as in Fig. 14, but the source redshift distribution is assumed to be higher, with $\left(z_{0}, \alpha, \beta\right)=$ $(0.9,2,1.5)$.

only slightly modified. The detailed analysis involving a marginalisation over $\Gamma$ and over the redshift distribution of the sources (constrained using photometric redshifts) is left for a forthcoming study. However, for the reasonable values of $\Gamma$, the degeneracy-breaking for the high $\Omega_{0}$ models is not affected by the present uncertainty on the redshift distribution of the sources. Our result is consistent with the rough guide given by the scaling $\sigma_{8} \Omega_{0}^{0.6} \propto z_{0}^{-0.5}$ (Jain \& Seljak 1997).

\section{Small scale signal}

Our correlation function measurements extend to much smaller scales than shown in the figures above. The limit is set only by the fact that we reject one member of all pairs closer than 3 arcsec. Figures 17 and 18 show the tangential, radial and total shear correlation functions. The pair separation bins are much smaller than in Figs. 5 and 6 , which explains why the error bars are larger. Even at the 


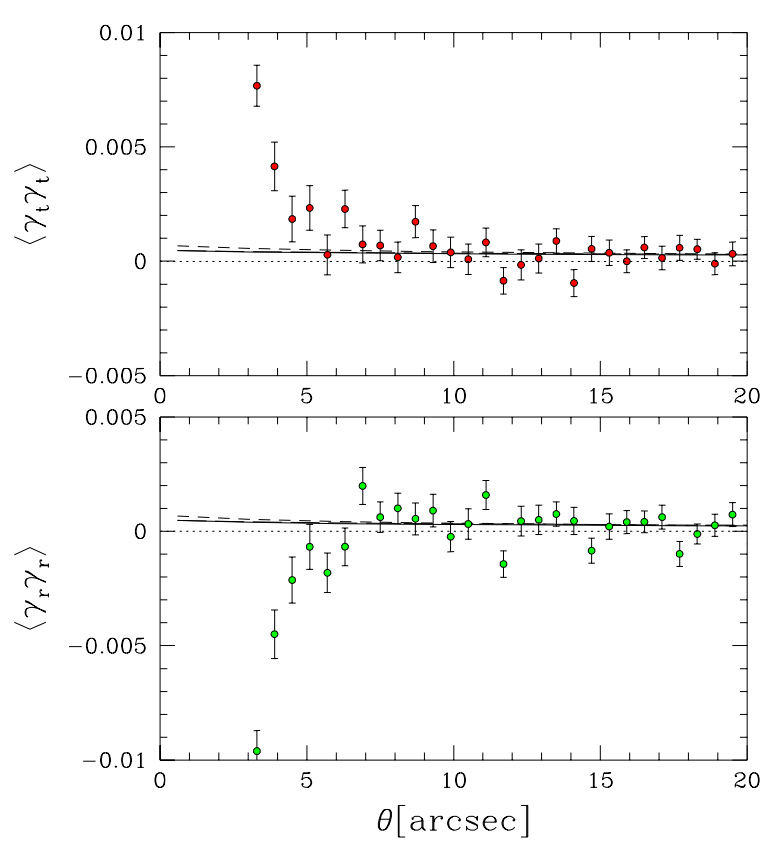

Fig. 17. Tangential (top panel) and radial (bottom panel) shear correlation functions $\left\langle\gamma_{\mathrm{t}} \gamma_{\mathrm{t}}\right\rangle_{\theta}$ and $\left\langle\gamma_{\mathrm{r}} \gamma_{\mathrm{r}}\right\rangle_{\theta}$ down to $3^{\prime \prime}$. The solid, long-dashed (hidden by the solid line) and short-dashed lines are predictions from the same models as in Fig. 3.

smallest scales, the shear correlation function $\langle\gamma \gamma\rangle_{\theta}$ is consistent with the model predictions.

The surprising result for the small scale correlations is the behavior of the tangential and radial shear correlation functions: at scales smaller than $5^{\prime \prime}$ we find an increased amplitude for $\left\langle\gamma_{\mathrm{t}} \gamma_{\mathrm{t}}\right\rangle_{\theta}$, and a negative $\left\langle\gamma_{\mathrm{r}} \gamma_{\mathrm{r}}\right\rangle_{\theta}$. Though surprinsing, a negative $\left\langle\gamma_{\mathrm{r}} \gamma_{\mathrm{r}}\right\rangle_{\theta}$ is not unphysical: for instance in Kaiser (1992) (Table 1), a shallow mass power spectrum $(n>-1)$ implies such an effect. In terms of halo mass profile, it corresponds to a projected profile steeper than -1.5 . However, regardless of the nature of this signal, it is important to note that this is a very small scale effect which has no impact on the statistics discussed in preceding sections. The contribution of the increased signal from $\left\langle\gamma_{\mathrm{t}} \gamma_{\mathrm{t}}\right\rangle_{\theta}$ to the variance at $1^{\prime}$ is less than $1 \%$; moreover since $\langle\gamma \gamma\rangle_{\theta}$ is not affected at all, the variance is also unaffected. As an explicit test, we checked that by removing one member of the pairs closer than $7^{\prime \prime}$ the measured signal in Figs. 3-6 is unchanged. In a similar cosmic shear analysis using the Red-sequence Cluster Survey (Gladders \& Yee 2000) another group finds a similar small scale behavior, though at lower statistical significance $(\mathrm{H}$. Hoekstra, private communication).

The cross-correlation $\left\langle\gamma_{\mathrm{t}} \gamma_{\mathrm{r}}\right\rangle_{\theta}$ vanishes down to $3^{\prime \prime}$, therefore no obvious systematic is responsible for this effect. The effect is unlikely to be caused by overlapping isophotes, or close neighbors effects because $\left\langle\gamma_{\mathrm{t}}\right\rangle_{\theta}^{2}<<$ $\left\langle\gamma_{\mathrm{t}} \gamma_{\mathrm{t}}\right\rangle_{\theta}$ : if it were a close neighbor alignment we would expect that $\left\langle\gamma_{\mathrm{t}}\right\rangle_{\theta}$ (the average tangential ellipticity for all the pair members in each pair separation bin $\theta$ ) carries all of the signal, which is not the case. In fact we find $\left\langle\gamma_{\mathrm{t}}\right\rangle_{\theta}^{2} \sim 0.2\left\langle\gamma_{\mathrm{t}} \gamma_{\mathrm{t}}\right\rangle_{\theta}$, which means that a close neighbor effect can hardly exceed $20 \%$ of the small scale signal.

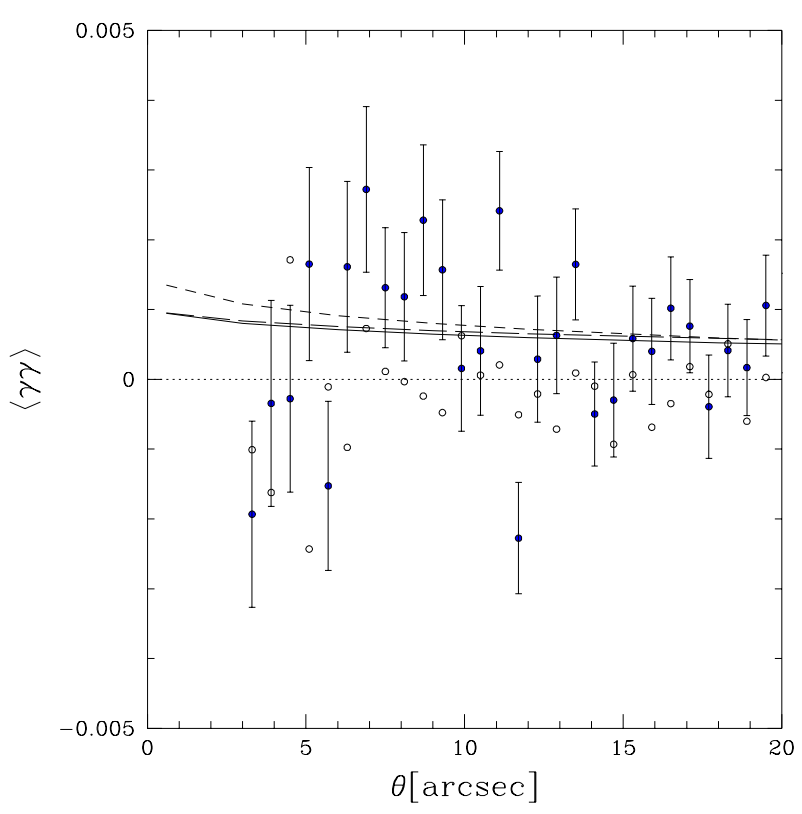

Fig. 18. Same as Fig. 17 but for the shear correlation function $\langle\gamma \gamma\rangle_{\theta}$. The open circles correspond to the cross-correlation $\left\langle\gamma_{\mathrm{t}} \gamma_{\mathrm{r}}\right\rangle$.

A forthcoming paper using the same data set will be devoted to the measurement of $E$ and $B$ modes (as defined in Crittenden et al. 2000a), and we will study this small scale signal in more detail. At this stage of the analysis we cannot exclude a possible residual systematic. However, a preliminary analysis shows that the $B$ mode down to $3^{\prime \prime}$ is much smaller than the $E$ mode, which is hard to understand if the signal comes entirely from residual systematics.

\section{Conclusion}

Using $6.5 \mathrm{deg}^{2}$ of the VIRMOS-Descart survey in progress at the CFHT, we were able to measure various 2-point correlation statistics of cosmic shear. The top-hat variance, the aperture mass statistic and different shear correlation functions gave consistent results over a wide range of scales. Further tests of the lensing origin of the signal exploiting the scalar nature of the gravitational potential were also convincingly verified. We demonstrated that the level of systematics, in particular the intrinsic alignment of galaxies, is likely to be small, and does not contribute to the signal for scales larger than $1^{\prime}$. For scales smaller than $1^{\prime}$ we have either ignored the measurements, or increased the error bars by the amount of signal found in the $B$-mode analysis which will be presented elsewhere. We believe that these results demonstrate the significance of our detection of shear correlations due to gravitational lensing. The quality of the data and the adequate size of our survey allow us to put some constrains on the cosmological models. We obtained tight constraints on the cosmological parameters $\Omega_{0}$ and $\sigma_{8}$ modulo, the uncertainties in the value of the power spectrum slope $\Gamma$ and in the source redshift distribution. We found that different values of $\Gamma$ significantly affects the degeneracy breaking in the $\Omega_{0}, \sigma_{8}$ plane. Only the use of external priors can allow 
robust and independent constraints on these parameters. A detailed analysis based on a marginalisation of $\Gamma$ and the observed redshift distribution (using photometric redshifts) will be done in a forthcoming paper. Nevertheless these results suggest that high precision measurements can be made with larger lensing surveys on a much larger set of cosmological parameters. Clearly, weak lensing surveys will play an important role in measuring the cosmological parameters together with Cosmic Microwave Background, Supernovae, and Large Scale Structures (Hu \& Tegmark 1999).

The final stage of the VIRMOS survey is to accomplish $16 \mathrm{deg}^{2}$ in patches of $4 \mathrm{deg}^{2}, 4$ colors each, thus allowing the possibility to use the photometric redshifts of the galaxies. The use of photometric (or spectroscopic) redshifts will be useful to put robust constraints on the cosmological parameters and improve the scientific interpretation of cosmic shear (e.g. doing tomography as in $\mathrm{Hu}$ 1999) but also to measure the intrinsic alignments itself (which can be used to constrain galaxy formation models for instance).

The constraints obtained so far are within a framework of structure formation through gravitational instability with Gaussian initial conditions and Cold Dark Matter. As the amount of observations increases and the measurement quality improves, the first hints of the shape of the power spectrum will be soon available. It opens new means of really testing the formation mechanisms of the largescale structure and the cosmological parameters beyond the standard model (Uzan \& Bernardeau 2000).

Over the last two years, we have seen the transition from the detection of the weak lensing signal to the first measurements of cosmological parameters from it. The agreement between theoretical predictions and observational results with such a high precision indicates that the measurement of cosmic shear statistics is becoming a mature cosmological tool. Many surveys are under way or scheduled for the next 5 years. They will use larger panoramic cameras than the CFH12K, and will cover solid angles 10 to 100 times wider than this work. The results of this work give us confidence that cosmic shear statistics will provide valuable measurements of cosmological parameters, probe the biasing of mass/light, produce maps of the dark matter distribution and reconstruct its power spectrum.

Acknowledgements. We are grateful to S. Colombi, U.-L. Pen, D. Pogosyan, S. Prunet, I. Szapudi and S. White for useful discussions related to statistics. We thank H. Hoekstra for sharing his results prior to publication. This work was supported by the TMR Network "Gravitational Lensing: New Constraints on Cosmology and the Distribution of Dark Matter" of the EC under contract No. ERBFMRX-CT97-0172, and a PROCOPE grant No. 9723878 by the DAAD and the A.P.A.P.E. We thank the TERAPIX data center for providing its facilities for the data reduction of $\mathrm{CFH} 12 \mathrm{~K}$ images.

\section{References}

Bartelmann, M., \& Schneider, P. 1999, A\&A, 345, 17

Bartelmann, M., \& Schneider, P. 2001, Phys. Rep., 340, 291
Bacon, D., Réfrégier, A., \& Ellis, R. 2000, MNRAS, 318, 625

Bertin, E., \& Arnouts, S. 1996, A\&A, 117, 393

Bernardeau, F., Van Waerbeke, L., \& Mellier, Y. 1997, A\&A, 322,1

Catelan, P., Kamionkowski, M., \& Blandford, R. 2000, MNRAS, 320, L7

Cuillandre, J.-C., Luppino, G., Starr, B., \& Isani, S. 2000, Proc. SPIE, 4008, 1010

Cohen, J. G., Hogg, D. W., Blandford, R., et al. 2000, ApJ, 538,29

Crittenden, R., Natarajan, P., Pen, U., \& Theuns, T. 2000a, [astro-ph/0009052]

Crittenden, R., Natarajan, P., Pen, U., \& Theuns, T. 2000b, [astro-ph/0012336]

Croft, R. A. C., \& Metzler, C. 2000, ApJ, 545, 561

Eisentsein, D., \& Zaldarriaga, M. 2001, ApJ, 546, 2

Erben, T., Van Waerbeke, L., Bertin, E., Mellier, Y., \& Schneider, P. 2001, A\&A, 366, 717

Heavens, A., Réfrégier, A., \& Heymans, C. 2000, MNRAS, 319, 649

Hu, W. 1999, ApJ, 522, 21

Gladders, M. D., \& Yee, H. K. C. 2000, The Toronto Red-Sequence Cluster Survey: First Results, to appear in Cosmic Evolution and Galaxy Formation: Structure, Interactions and Feedback, [astro-ph/0002340]

Hu, W., \& Tegmark, M., ApJ, 514, 65

Jain, B., \& Seljak, U. 1997, ApJ, 484, 560

Jain, B., Seljak, U., \& White, S. 2000, ApJ, 530, 547

Kaiser, N. 1992, ApJ, 388, 272

Kaiser, N. 1998, ApJ, 498, 26

Kaiser, N., Squires, G., Fahlman, G., \& Woods, D. 1994, in Clusters of Galaxies, ed. F. Durret, A. Mazure, \& J. Tran Thanh Van (Éditions Frontières Gif-sur-Yvette), 269

Kaiser, N., Squires, G., \& Broadhurst, T. 1995, ApJ, 449, 460

Kaiser, N., Wilson, G., \& Luppino, G. 2000 [astro-ph/0003338]

Le Fèvre, O., Vettolani, P., Maccani, D., et al. 2001, Proc. of the ESO/ECF/STSCI, Deep Surveys workshop, Garching Oct. 2000 (Springer)

Landolt, A. U. 1992, AJ, 104, 340.

Luppino, G., \& Kaiser, N. 1997, ApJ, 475, 20

Maoli, R., Van Waerbeke, L., Mellier, Y., et al. 2001, A\&A, 368,766

Mellier, Y. 1999, ARAA, 37, 127

Miralda-Escudé, J. 1991, ApJ, 380, 1

Moessner, R., Jain, B., \& Villumsen, J. V. 1998, MNRAS, 294, 291

Peacock, J. A., \& Dodds, S. J. 1996, MNRAS, 280, L9

Pen, U. L., Lee, J., \& Seljak, U. 2000, ApJ, 543, L107

Rhodes, J., Réfrégier, A., \& Groth, E. 2001, ApJ, 552, 85

Schneider, P., Van Waerbeke, L., Jain, B., \& Kruse, G. 1998, MNRAS, 296, 873

Uzan, J. P., \& Bernardeau, F. 2000 [hep-ph/0012011]

Van Waerbeke, L., Bernardeau, F., \& Mellier, Y. 1999, A\&A, 342,15

Van Waerbeke, L., Mellier, Y., Erben, T., et al. 2000, A\&A, 358,30

Van Waerbeke, L., Hamana, T., Scoccimarro, R., Colombi, S., \& Bernardeau, F. 2001, MNRAS, 322, 918

Villumsen, J. 1996, MNRAS, 281, 369

Wilson, G., Kaiser, N., Luppino, G. A., \& Cowie, L. L., ApJ, submitted [astro-ph/0008504]

Wittman, D. M., Tyson, A. J., Kirkman, D., Dell'Antonio, I., \& Bernstein, G. 2000, Nature, 405, 143 